\title{
Variational quantum Monte Carlo ground state of GaAs
}

\author{
H. Eckstein and W. Schattke \\ Institut für Theoretische Physik, Universität Kiel, D-24098 Kiel, Germany \\ M. Reigrotzki and R. Redmer \\ Fachbereich Physik, Universität Rostock Universitätsplatz 3, D-18051 Rostock, Germany
}

(Received 18 March 1996)

\begin{abstract}
Variational quantum Monte Carlo calculations are reported for the bulk GaAs semiconductor in order to present values for the ground-state energy, the lattice constant, the bulk modulus, and some derived properties. The statistical accuracy is significantly higher than the remaining differences to the experimental values, especially for the total-energy upper bound. The agreement with experiment is satisfactory. The results are also compared with those of density functional calculations. The accuracy of our results is comparable to the best of those calculations.
\end{abstract}

PACS Numbers: $71.15 \mathrm{Nc}, 61.50 \mathrm{Lt}$

\section{INTRODUCTION}

In comparison with the widely accepted and extensively applied local density functional technique (LDA), the quantum Monte Carlo methods (QMC) represent rather new developments for the ground-state determination of solids. 0 Especially the variational quantum Monte Carlo method (VQMC) is attractive, because the quality of the approximation is controlled by the property of yielding an upper bound, i.e., by the simple rule: the lower the energy the better the wave function. The statistical error of an expectation value can be safely estimated. It behaves favorably near an eigenvalue where the variance should vanish. Thus this method is really ab initio, not relying on any uncontrolled quantity, such as an unknown correlation functional in the case of LDA. This aspect seems to be actually important, because the-detailed results of the density functional theory (DFT) in fact depend sensitively on the choice of this functional.10 13 Furthermore, in the past the role of Monte Carlo simulations for homogeneous systems was to determine the correlation energy to be adapted as functional for inhomogeneous LDA calculations. 3 Thus today's QMC calculations on inhomogeneous systems may also be valued by the DFT as it may lead to an improved functional.

The first paper to apply VQMC to a solid of nonlight elements was presented by Fahy, Wang, and Louid dealing with the ground-state determination of diamond, graphite, and silicon. They proved that such a calculation is feasible if one relies on a pseudopotential to replace the inner shells' contribution. Different from the low atomic number systems 4.6 an uncertainty thereby enters into this technique by the construction of the pseudopotential itself. In order to treat the system on an equal footing with LDA, it is adequate to use the nonlocal $a b$ initio potential developed by Hamann 14

In this paper we apply the VQMC method to the compound semiconductor GaAs to calculate the ground-state properties. We closely rely on our former paper on lithium in treating the solid by simulating a finite system and in treating various other calculational details. Atomic simulations are carried through to obtain the binding energy with respect to these reference values. Hartree-Fock calculations have been peformed to extract the correlation energy. The inner shell electrons are included within a nonlocal pseudopotential for the valence electrons.

In Sec. [1] we briefly expose the theoretical method used by VQMC. The results are discussed in Sec. III.

\section{METHOD}

The Monte Carlo algorithm calculates expectation values of the form

$$
\langle f\rangle=\frac{\int f(x) p(x) d x}{\int p(x) d x},
$$

where the integration variable $x$ denotes typically several hundred single variables. The error is of purely statistical nature and depends on the number of simulation points as well as on the variance and the autocorrelation function of $f$. The quantum mechanical expectation value of an observable $B$ is calculated by setting 


$$
p=|\psi|^{2}, \quad f=(B \psi) / \psi .
$$

The smaller the variance of $(B \psi) / \psi$, the smaller the statistical error of the calculation, which can be taken as a measure for the many-body wave function $\psi$ to be an eigenfunction of $B$.

In the present approach we simulated $N$ valence electrons in a supercell containing a limited number of crystal unit cells. We sampled systems of 4 and $32 \mathrm{GaAs}$ unit cells to test for convergence. Only the results for the large system with 256 electrons are described here. To model the solid, periodic boundary conditions are assumed, which affect the Hamiltonian and the wave function as well. The Hamiltonian

$$
H=T+E_{c}+E_{\mathrm{loc}}+E_{\mathrm{nloc}}
$$

consists of the kinetic energy $T$, the Coulomb energy $E_{c}$, and the local part $E_{\text {loc }}$ as well as the nonlocal part $E_{\text {nloc }}$ of the additional electron-ion pseudopotential, which is a generalized norm-conserving ab initio atomic pseudopotential.14 The Coulomb energy includes the nucleon-nycleon, the electron-ion, and the electron-electron part. It is calculated by means of the Ewald summation technique.15 The local part of the pseudopotential can be decomposed into a sum over atoms $\alpha$ at site $\mathbf{R}_{\alpha}$,

$$
E_{\mathrm{loc}}=\sum_{\alpha, \mathbf{R}_{\alpha}, s, i} V_{\mathrm{loc}, \alpha}\left(\mathbf{r}_{i}^{s}\right)
$$

with $\mathbf{r}_{i}^{s}$ being the position relative to $\mathbf{R}_{\alpha}$ of the $i$ th electron of spin $s$. The respective sum for the nonlocal pseudopotential contains a summation over angular momenta $l$, upon which the wave function is projected:

$$
V_{\mathrm{nloc}, \alpha}\left(\mathbf{r}_{i}^{s}=\mathbf{r}\right)=\sum_{l} V_{\alpha, l}(r) \frac{Y_{l 0}(0,0) \int_{r^{\prime}=r} Y_{l 0}^{*}\left(\Omega_{r^{\prime}}\right) \psi\left(\ldots, \mathbf{r}_{i}^{s}=\mathbf{r}^{\prime}, \ldots\right) d \Omega_{r^{\prime}}}{\psi\left(\ldots, \mathbf{r}_{i}^{s}=\mathbf{r}, \ldots\right)} .
$$

The spherical angle $\Omega_{r^{\prime}}$ is referred to the direction of $\mathbf{r}$ in an arbitrary but fixed polar coordinate system. The projection operator demanding high numerical efforts needs only to be calculated where the radial pseudopotential $V_{\alpha, l}\left(r_{i}^{s}\right)$ is nonzero, i.e., when $\mathbf{r}_{i}^{s}$ lies within the radius of the atomic pseudopotential. The integration is done as a sum over a grid of four points on the sphere that is exact for $l \leq 2.6$

The wave function consists of a product of two Slater determinants and a Jastrow factor:

$$
\Psi\left(\mathbf{r}_{1}, \ldots, \mathbf{r}_{N}\right)=D^{\uparrow}\left(\mathbf{r}_{1}, \ldots, \mathbf{r}_{N / 2}\right) D^{\downarrow}\left(\mathbf{r}_{N / 2+1}, \ldots, \mathbf{r}_{N}\right) \exp \left(-\sum_{i<j} u\left(r_{i j}\right)\right) .
$$

The exponent of the Jastrow factor is chosen in the usual form for solids:

$$
u(r)=\frac{A}{r}\left(1-e^{-r / F}\right),
$$

where $A$ is a variational parameter. For a given $A$ the value of $F$ is fixed by the cusp condition, which removes the Coulomb singularity. The Slater determinants $D^{\uparrow}, D^{\downarrow}$ consist of parametrized hybrid bonds in the tetrahedral directions $\vec{\tau}$ from the arsenic ion

$$
\begin{aligned}
\phi(\mathbf{r}) & =\phi_{\mathrm{hyb}}^{\mathrm{As}}+\beta \phi_{\mathrm{hyb}}^{\mathrm{Ga}}, \\
\phi_{\mathrm{hyb}}^{\mathrm{As}}(\mathbf{r}) & =\gamma^{\mathrm{As}} \phi_{s}^{\mathrm{As}}\left[\left(\mathbf{r}-\mathbf{R}_{\mathrm{As}}\right) / \zeta_{s}^{\mathrm{As}}\right]+\sum_{i} \tau_{i} \phi_{p_{i}}^{\mathrm{As}}\left[\left(\mathbf{r}-\mathbf{R}_{\mathrm{As}}\right) / \zeta_{p}^{\mathrm{As}}\right], \\
\phi_{\mathrm{hyb}}^{\mathrm{Ga}}(\mathbf{r}) & =\gamma^{\mathrm{Ga}} \phi_{s}^{\mathrm{Ga}}\left[\left(\mathbf{r}-\mathbf{R}_{\mathrm{Ga}}\right) / \zeta_{s}^{\mathrm{Ga}}\right]-\sum_{i} \tau_{i} \phi_{p_{i}}^{\mathrm{Ga}}\left[\left(\mathbf{r}-\mathbf{R}_{\mathrm{Ga}}\right) / \zeta_{p}^{\mathrm{Ga}}\right],
\end{aligned}
$$

where $\beta, \gamma$, and $\zeta$ are variational parameters and $i$ runs over the Cartesian components $(x, y, z)$. The quantities $\zeta$ describe the contraction of the wave functions as usual for atomic orbitals in solids and reduce the additional extent introduced by the Jastrow factor. The coefficients $\gamma$ and $\beta$ represent the adjustable parameters for building hybrid bonds. The wave function is automatically normalized within the QMC formalism.

For the ratios $\Psi_{\text {new }} / \Psi_{\text {old }}$ of the random walk and in the projection operator as well as in the parts contributing to the kinetic energy, the inverse matrices of the Slater matrices are conveniently determined numerically by the ShermanMorrison-Woodbury formula.The periodic boundary conditions with respect to the wave functions are controlled by Green's relation $-\int \Psi^{*} \Delta \Psi=\int|\nabla \Psi|^{2}$. 
The minimization over the huge parameter space is done as follows. Several hundreds of parameter sets in the region of the guessed minimum are chosen from a multidimensional Gaussian distribution. The total-energy expectation values weighted with their reciprocal statistical errors are fit into a multidimensional quadratic function. If the minimum of the fit lies within the region of the chosen statistical ensemble, a few parameter sets are selected along the minimum line of the fit with an increased number of simulation points. This calculation along the minimum line is statistically independent of the prior calculation with the large number of parameter sets and therefore not biased as it would be, if the absolute minima of several calculations were explicitly taken.

\section{RESULTS}

At first, a minimization for the ground-state energies of the gallium and the arsenic atom has been carried through. Table $\llbracket$ gives an overview of the obtained quantities. The total energies are lower than the experimental values, which are the sums of the ionization potentials of the valence electrons. Only the valence electrons have been simulated in this approach. The differences are supposed to indicate the error from the frozen-core approximation using pseudopotentials, also apparent in the first ionization potentials. As expected, the $\zeta$ parameters all result in 1.0 from the minimization of the Hartree-Fock approach. Introducing the Jastrow factor we observe compression of the atomic orbitals even in the free atom. To deduce the constituents of the total energy, we made a Hartree-Fock simulation. The first ionization potentials are obtained from the approximation that the wave function is represented by the same parametrization scheme, i.e., by that of the neutral atom.

TABLE I. Ground-state energies in eV of the gallium and arsenic atom from a variational QMC calculation: the first two lines are from Hartree-Fock calculations, the Hartree part of the electron-electron interaction is externally calculated, the exchange energy is the difference between the Hartree-Fock interaction energy and that Hartree part, the correlation energy is the difference between the total energy and the Hartree-Fock minimum total energy; the experimental total energy is the sum over the ionization potentials of the simulated electrons; the last line gives the first ionization potential.

\begin{tabular}{|c|c|c|c|c|}
\hline Energy & Ga expt. & Ga VQMC & As expt. & As VQMC \\
\hline 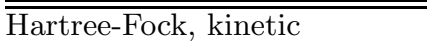 & & $20.28 \pm 0.01$ & & $54.79 \pm 0.03$ \\
\hline Hartree-Fock, potential & & $-77.04 \pm 0.01$ & & $-222.92 \pm 0.04$ \\
\hline Hartree, electron-electron & & $42.08 \pm 0.04$ & & $134.3 \pm 0.7$ \\
\hline Exchange, electron-electron & & $-16.21 \pm 0.04$ & & $-37.3 \pm 0.8$ \\
\hline Correlation, electron-electron & & $-1.250 \pm 0.008$ & & $-2.00 \pm 0.02$ \\
\hline Total & -57.21 & $-58.000 \pm 0.003$ & -169.554 & $-170.134 \pm 0.007$ \\
\hline Ionization & 5.999 & $6.10 \pm 0.02$ & 9.81 & $10.38 \pm 0.02$ \\
\hline
\end{tabular}

TABLE II. Ground-state energies in eV per unit cell of gallium arsenide from a variational QMC calculation; $E_{\text {kin }}$ and $E_{\text {pot }}$ are produced by Hartree-Fock calculations, $E_{\text {corr }}$ is the difference between $E_{\text {tot }}$ and the Hartree-Fock minimum total energy, $E_{\text {coh }}$ is the difference between $E_{\text {tot }}$ and the atomic energies, the experimental cohesive energy has been taken from Ref. 17, the experimental total energy is the sum of the cohesive energy and the atomic total energies; $a_{0}$ denotes the lattice constant of the zinc-blende crystal and $B$ is the bulk modulus at temperature $T=0 ;$ LDA as well as the Becke and the Perdew gradient corrections (BP) are from density functional calculations of Ref. 11.

\begin{tabular}{lccc}
\hline \hline GaAs & Expt. & VQMC & LDA \\
\hline \hline$E_{\text {kin }}$ & & $84.6 \pm 0.2$ & \\
$E_{\text {pot }}$ & & $-311.3 \pm 0.3$ & \\
$E_{\text {corr }}$ & $-6.42 \pm 0.2$ & \\
$E_{\text {tot }}$ & $-233.43 \pm 0.09$ & $-233.04 \pm 0.08$ & -8.16 \\
$E_{\text {coh }}$ & $-6.67 \pm 0.09$ & $-4.9 \pm 0.2$ & 10.41 \\
$a_{0}$ in a.u. & 10.6830 & $10.69 \pm 0.1$ & -6.45 \\
$B$ in kbar & 756 & $786 \pm 100$ & 10.70 \\
\hline \hline
\end{tabular}


Figure 1 shows the ground-state total energy of the gallium arsenide solid with 256 electrons as a function of the lattice constant, minimized with respect to all wave-function parameters. The curve is the quadratic function obtained from a weighted Gaussian fit of the data points. Table II shows the obtained quantities in comparison with the experimental ones and density functional calculations.11 The best agreement with the experiment is found for the total energy. The pseudopotentials give excellent results in this approach of parametrized linear combination of atomic orbitals wave functions even with the semilocal approximation. Differences in the cohesive energy originate from underestimating the atomic total energies of As and Ga owing to deficiencies of the pseudopotential in the atomic calculation. The lattice constant agrees very well with the experimental value, although this quantity statistically arises with a somewhat larger uncertainty than the total energy. The respective error given in Table II is an upper bound estimated by just considering the fit in Fig. 1.

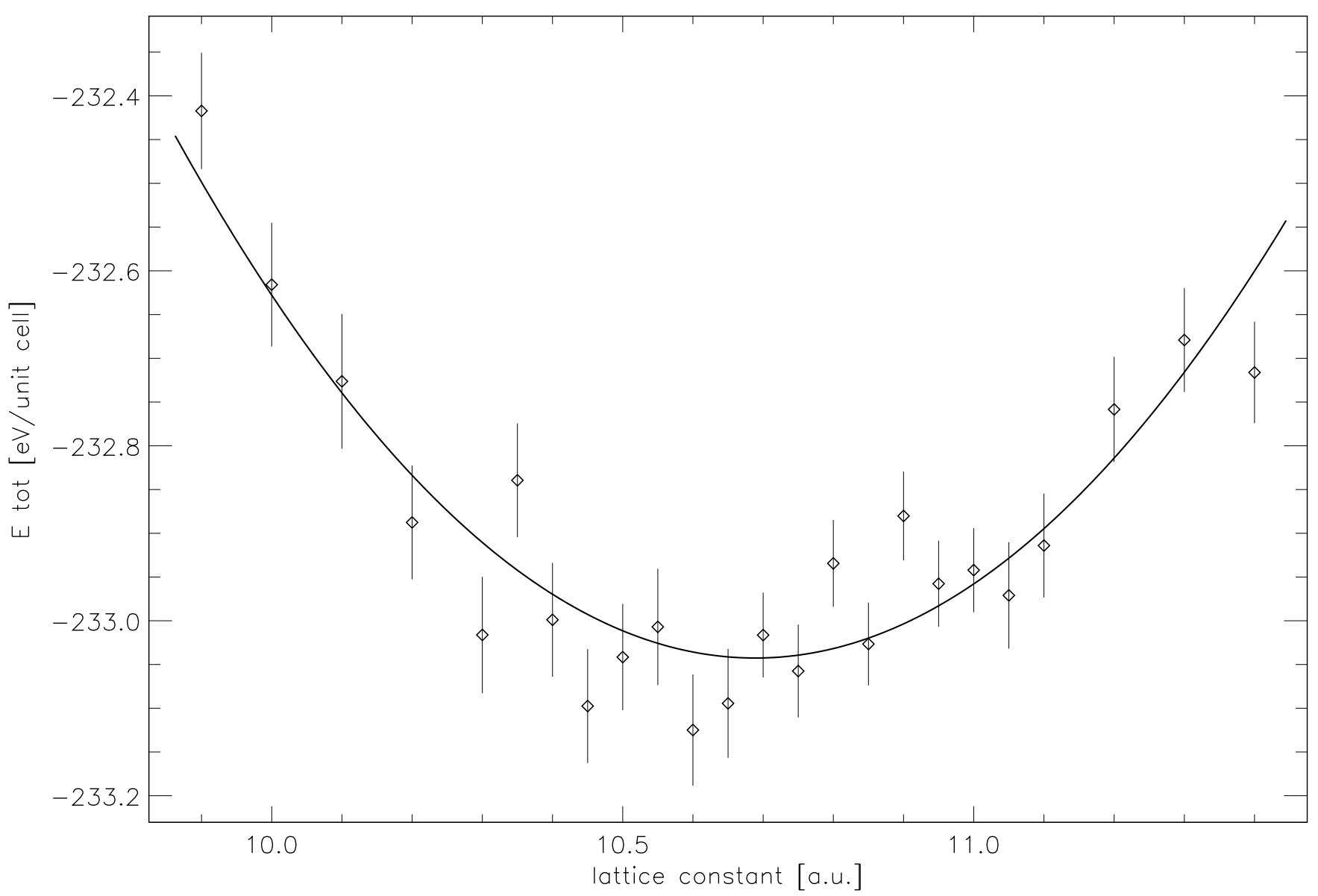

FIG. 1. Total energy vs lattice constant of gallium arsenide from a VQMC calculation including 256 valence electrons; the curve is a quadratic fit.

The bulk modulus is obtained slightly too high, which indicates that lattice constants in the far neighborhood of the minimum are treated worse in the minimization procedure than those in the close neighborhood. This may be understood by observing that the minimum line is the result of the multidimensional quadratic fit, i.e., a straight line through the parameter space. In the far neighborhood of the global minimum this restriction could yield nonminimum parameters. The parts the total energy is composed of, as given in Table [1], are calculated in the minimum of the Hartree-Fock approach. The minimization was more difficult in this case, because the Coulomb cusp leads to a larger variance in the total energy. The difference between the results of the Hartree-Fock ansatz and the Jastrow-Slater ansatz, which defines the correlation energy, shows no statistically significant behavior in the simulated range of the lattice constant. Therefore the value of $-6.42 \mathrm{eV}$ per unit cell does not vary much near the ground-state density. The statistical data set for the correlation yields a straight regression line, the energy increasing with decreasing electron density with a slope of $0.9 \pm 0.9 \mathrm{eV}$ per $r_{s}$. A measure for the ionicity is obtained by counting the electron visits to specific Voronoi polyhedra during a run. The polyhedra are constructed according to a partition of the crystal in which lattice sites are added at the empty tetrahedral positions and at the cube center, the so-called empty 
spheres' positions in zinc-blende muffin-tin calculations. As a result the occupied As, occupied Ga, unoccupied As, and unoccupied Ga sites show charges of 3.9, 2.2, 0.9, and 1.0 electrons, respectively. Thus, both ions are positively charged the separated electrons extending into the interstitial space. The covalent bond charge was estimated by placing the centers of Voronoi polyhedra upon the bonds of the above lattice so that the polyhedra cover the bond region between sites that are occupied or empty. A slight charge accumulation appears on the occupied Ga-As bond of 0.13 electron per bond in addition to the charge which arises by the spherically symmetric electron distribution of the neutral atoms.

Summarizing the obtained results, the good agreement with experiment puts the variational quantum Monte Carlo method into competition with current density functional techniques as far as the absolute ground-state quantities, calculated here, are concerned. The minimum principle guarantees an additional reliability absent in DFT, which in contrast bears an uncertainty because of the, in some respects, arbitrary choice of the density functional. As the latter is able to treat more complicated solids, the comparison with QMC calculations on the basis of simple systems may lead to a selection and adjustment of suitable functionals.

\section{ACKNOWLEDGMENTS}

We are indebted to Dr. E. Pehlke for various discussions on the topic of DFT. The work was supported by the Deutsche Forschungsgemeinschaft under grants No. Re 882/6-1 and No. Scha 360/8-1.

${ }^{1}$ W. L. McMillan, Phys. Rev. 138, A442 (1965).

${ }^{2}$ D. M. Ceperley, G. V. Chester, and M. H. Kalos, Phys. Rev. B 16, 3081 (1977).

${ }^{3}$ D. M. Ceperley and B. J. Alder, Phys. Rev. Lett. 45, 566 (1980).

${ }^{4}$ D. M. Ceperley and B. J. Alder, Phys. Rev. B 36, 2092 (1987).

${ }^{5}$ S. Fahy, X. W. Wang, and S. G. Louie, Phys. Rev. Lett. 61, 1631 (1988); Phys. Rev. B 42, 3503 (1990), and references therein.

${ }^{6}$ G. Sugiyama, G. Zerah, and B. J. Alder, Physica A 156, 144 (1989).

${ }^{7}$ H. Eckstein and W. Schattke, Physica A 216, 151 (1995).

${ }^{8}$ A. C. Cancio and Y.-C. Chang, Phys. Rev. B 47, 13246 (1993).

${ }^{9}$ G. Rajagopal et al., Phys. Rev. B 51, 10591 (1995).

10 A. García et al., Phys. Rev. B 46, 9829 (1992).

${ }^{11}$ G. Ortiz, Phys. Rev. B 45, 11328 (1992).

12 C. Filippi, D. J. Singh, and C. J. Umrigar, Phys. Rev. B 50, 14947 (1994).

${ }^{13}$ M. Causà and A. Zupan, Chem. Phys. Lett. 220, 145 (1994).

${ }^{14}$ D. R. Hamann, Phys. Rev. B 40, 2980 (1989); D. R. Hamann, M. Schlüter, and C. Chiang, Phys. Rev. Lett. 43, 1494 (1979).

15 A. Sugiyama, J. Phys. Soc. Jpn. 53, 1624 (1984).

${ }^{16}$ V. I. Lebedev, USSR Comput. Math. Math. Phys. 16, 10 (1976).

17 J. R. Arthur, J. Phys. Chem. Solids 28, 2257 (1967). 